\title{
Identification of Ventricular Tachycardia Using Intracardiac Electrograms: A Comparison of Unipolar Versus Bipolar Waveform Analysis
}

\author{
SAUL E. GREENHUT, * LORENZO A. DICARLO, * * JANICE M. JENKINS, * \\ ROBERT D. THRONE, ${ }^{*}$ and STUART A. WINSTON** \\ From * The Department of Electrical Engineering and Computer Science and the Bioengineering \\ Program, University of Michigan, Ann Arbor, Michigan, and * The Cardiac Electrophysiology \\ Laboratory, St. Joseph Mercy Hospital of the Catherine McAuley Health Center, and the \\ University of Michigan Medical School, Ann Arbor, Michigan
}

GREENHUT S.E., ET AL. Identification of Ventricular Tachycardia Using Intracardiac Electrograms: A Comparison of Unipolar Versus Bipolar Waveform Analysis. Implantable antitachycardia devices suffer a high false-positive rate of delivery of therapy because current detection schemes based upon ventricular rate and rate variations are excessively sensitive at the cost of specificity. Several methods have been proposed for providing complementary information derived from morphologic analysis of intraventricular electrograms in order to increase specificity. The majority of these techniques have utilized bipolar electrogram analysis to detect changes in ventricular activation indicative of ventricular tachycardia. Whether bipolar or unipolar intracardiac electrogram analysis might be preferred for discriminating ventricular tachycardia from sinus rhythm has not been determined. In this study, a previously demonstrated method for identification of ventricular tachycardia using intracardiac electrograms, correlation waveform analysis, was used to analyze both unipolar and bipolar signals during sinus rhythm and ventricular tachycardia recorded during electrophysiology studies of 15 patients with inducible sustained monomorphic ventricular tachycardia. Correlation waveform analysis consistently discriminated between all depolarizations during ventricular tachycardia in 14/15 patients (93\%) using either electrogram configuration; 13 of the 14 patients were common to both groups. Of these patients, 8/15 $(53 \%)$ had greater separation between sinus rhythm and ventricular waveforms with bipolar electrogram analysis while $7 / 15$ (47\%) had greater separation with unipolar electrogram analysis. We conclude that morphologic analysis of unipolar and bipolar electrograms may be equally effective in distinguishing ventricular tachycardia from sinus rhythm. For individual patients, either a unipolar or bipolar ventricular configuration may be preferable, and should be chosen on a patient-specific basis during electrophysiology study prior to antitachycardia device implantation. (PACE, Vol. 14, March 1991)

ventricular tachycardia detection, correlation waveform analysis, unipolar ventricular electrogram, waveform morphology

\section{Introduction}

Appropriate treatment of ventricular tachycardia by antitachycardia devices depends upon

\footnotetext{
This work was partially sponsored by NSF grant No. BCS8909042 and a grant from Telectronics, Inc.

Address for reprints: Lorenzo A. DiCarlo, M.D., Catherine McAuley Health Center, Reichert Health Bldg., R-3003, P.O. Box 994, Ann Arbor, MI 48106. Fax: (313) 434-0141.

Received May 11, 1990; revision September 13, 1990; Accepted December 10, 1990.
}

accurate discrimination of this tachyarrhythmia from supraventricular tachycardias or sinus tachycardia. Currently, available devices have relied predominantly upon heart rate and rate variations to differentiate such dysrhythmias. ${ }^{1-10}$ Devices that depend upon such algorithms have been shown to have major limitations, however. High false-positive rates have been reported, resulting in delivery of inappropriate and potentially lethal therapy. ${ }^{11-13}$

Complementary algorithms that include analysis of intraventricular electrogram morphology 
have been proposed as a means of improving the accuracy of tachycardia discrimination. Among these, correlation waveform analysis has been shown to be effective in detecting ventricular tachycardia in patients with a normal QRS as well as chronic bundle branch block and nonspecific intraventricular conduction delays during sinus rhythm. ${ }^{14-16}$ Hallmarks of this analytic method include its lack of dependence upon electrogram amplitude and baseline fluctuations.

The majority of studies to date, however, have been limited to the analysis of bipolar intraventricular electrograms. Whether analysis of unipolar intraventricular electrograms might increase the accuracy or reliability of ventricular tachycardia detection by methods such as correlation waveform analysis has not been determined. The purpose of this study, therefore, was to assess the impact of unipolar versus bipolar intraventricular electrogram analysis upon the discrimination of ventricular tachycardia from sinus rhythm.

\section{Methods}

\section{Electrophysiology Study}

Electrograms were recorded during routine cardiac electrophysiological studies for the evaluation and treatment of sustained ventricular tachycardia. Our technique has been described previously. ${ }^{14,15}$ The patient population consisted of 14 men and 1 woman with ages $24-86$ years (mean $=61$ years). Fourteen of 15 patients had coronary artery disease with previous myocardial infarction. Patient characteristics are summarized in Table I. Three 6 French quadripolar electrode catheters with an interelectrode distance of $1 \mathrm{~cm}$ (catalog \#006245 from USCI Division, C.R. Bard Inc., Billerica, MA, USA) were introduced and advanced under fluoroscopic guidance. The three ring electrodes are cylinders $2 \mathrm{~mm}$ in diameter and $2 \mathrm{~mm}$ in length. The tip electrode consists of a half-sphere, diameter $2 \mathrm{~mm}$, attached to a cylinder of diameter $2 \mathrm{~mm}$ and length $1 \mathrm{~mm}$. The resulting surface area of each platinum ring and tip electrode is $12.6 \mathrm{~mm}^{2}$

One electrode catheter was positioned in the high right atrium or right atrial appendage. Two separate electrode catheters were positioned in the right ventricular apex, for pacing and record- ing respectively, in each case from the distal pair of electrodes. In addition to intracardiac atrial and ventricular electrograms, electrocardiographic leads $V_{1}$, I, and III were recorded continuously during the study.

Unipolar electrograms were recorded using the distal right ventricular apex electrode as the exploring electrode with a heparinized $0.9-\mathrm{mm}$ guidewire (Cordis Corporation, Miami, FL, USA) inserted into the 8 French venous sidearm sheath serving as the indifferent electrode. The guidewire was positioned in the right femoral vein extending into the right iliac and distal $5 \mathrm{~cm}$ of the inferior vena cava. The guidewire was continuously flushed with heparinized saline. Bipolar electrograms were recorded simultaneously from the distal pair of electrodes of the right ventricular apex recording catheter. At the end of each procedure, the guidewire and sheath were carefully examined and the absence of thrombus formation was confirmed in all cases.

\section{Data Acquisition}

Immediately prior to programmed stimulation, a 12-lead electrocardiogram was recorded during sinus rhythm. Unipolar and bipolar intraventricular electrograms were recorded. on FM magnetic tape (Hewlett-Packard 3968A, San Diego, CA, USA) after signal amplification and filtering of 1-500 Hz (Honeywell Electronics-For-Medicine, PPG Hellige, Freiburg, West Germany) during electrophysiological study. Tape speed was $3^{\frac{3}{4}}$ in $/ \mathrm{s}(9.5 \mathrm{~cm} / \mathrm{s})$ resulting in a bandwidth of $0-1250$ $\mathrm{Hz}$. Amplifier gain and filter settings were held constant during the entire recording procedure.

Passages of unipolar and bipolar intraventricular electrograms during sinus rhythm and sustained monomorphic ventricular tachycardia (> $30 \mathrm{~s}$, or terminated $\leq 30 \mathrm{~s}$ because of hemodynamic collapse) were concurrently digitized offline on an IBM-PC/AT compatible microcomputer (Advanced Logic Research 386/2, Irvine, CA, USA). A data acquisition system (Qua-Tech SAC-12, Akron, OH, USA), with a CODAS (Dataq Instruments, Akron, OH, USA) waveform display and storage system, was employed to digitize data at a frequency of $1000 \mathrm{~Hz} / \mathrm{channel}$. Tracing of both unipolar and bipolar electrograms and a surface electrocardiogram were obtained during digitiza- 
Table I.

Patient Information

\begin{tabular}{ccccccccc}
\hline Patient & Sex & Age & $\begin{array}{c}\text { Drug During } \\
\text { EPS }\end{array}$ & $\begin{array}{c}\text { SR } \\
\text { Morphology }\end{array}$ & $\begin{array}{c}\text { VT } \\
\text { Morphology }\end{array}$ & $\begin{array}{c}\text { Heart } \\
\text { Disease }\end{array}$ & Unipolar & Bipolar \\
\hline 1 & M & 79 & None & Normal & LBB-S & CAD & + & + \\
2 & M & 45 & Am, Pro & IVCD & RBB-S & CAD & + & + \\
3 & M & 67 & Dig & RBBB & LBB-S & CAD & + & + \\
4 & M & 76 & None & IVCD & LBB-S & CAD & + & + \\
5 & M & 73 & None & RBBB & RBB-S & CAD & + & + \\
6 & M & 53 & None & IVCD & RBB-S & CAD & + & + \\
7 & M & 24 & Iso & IVCD & RBB-S & None & + & + \\
8 & M & 61 & None & Normal & LBB-1 & CAD & + & + \\
9 & M & 36 & None & IVCD & RBB-S & CAD & + & + \\
10 & F & 63 & Qun & Normal & LBB-S & CAD & - & + \\
11 & M & 48 & None & RBBB & LBB-I & CAD & + & + \\
12 & M & 63 & En, Mex & IVCD & LBB-S & CAD & + & + \\
13 & M & 86 & None & RBBB & LBB-I & CAD & + & + \\
14 & M & 74 & Pro & Normal & RBB-S & CAD & + & + \\
15 & M & 63 & Qun & Normal & RBB-S & CAD & + & + \\
\hline
\end{tabular}

EPS = electrophysiology study; $\mathrm{SR}=$ sinus rhythm, VT = ventricular tachycardia; $\mathrm{Am}=$ amiodarone, Dig $=$ digoxin, En $=$ encainide, Iso = isoproterenol, Mex = mexiletine, Pro = procainamide, Qun = quinidine; LBBB = left bundle branch block, IVCD = intraventricular conduction delay (QRS duration $\geq 120 \mathrm{~ms}$ ), RBBB = right bundle branch block, LBB $=$ left bundle branch configuration, $\mathrm{RBB}=$ right bundle branch configuration, $\mathrm{I}=$ inferior axis, $\mathrm{S}=$ superior axis; $\mathrm{CAD}=$ coronary artery disease; + indicates complete separation of ventricular tachycardia and sinus rhythm $\eta$ ranges for lead configuration, - indicates no complete separation.

tion using strip chart recorders (Gould Brush 481, Gould, Cleveland, OH, USA). The digitized versions of these signals were simultaneously viewed on the computer monitor.

\section{Method of Analysis}

For each patient, a 30-second passage of sinus rhythm was digitized using unipolar and bipolar electrogram configurations. A second passage consisting of 11-30 s (mean = 18.7 s) of ventricular tachycardia was also recorded for each patient using both intraventricular lead configurations. A ventricular depolarization template was created for each patient for each lead configuration by signal averaging the first four beats of sinus rhythm. The remainder of this sinus rhythm passage and the entire ventricular tachycardia recording were subsequently analyzed using the appropriate unipolar or bipolar template.

Correlation waveform analysis, as previously described for ventricular bipolar ${ }^{14}$ and atrial bipolar electrogram analysis, ${ }^{15}$ was used as the method of comparison between sinus rhythm and ventricular tachycardia. Briefly, the technique of correlation waveform analysis provides a measure of the relative shape similarity between two waveforms. The correlation coefficient $(\rho)$ is defined as follows:

$$
\rho=\frac{\sum_{i=1}^{N}\left(T_{i}-\bar{T}\right)\left(S_{i}-\bar{S}\right)}{\sqrt{\sum_{i=1}^{N}\left(T_{i}-\bar{T}\right)^{2} \sum_{i=1}^{N}\left(S_{i}-\bar{S}\right)^{2}}}
$$

where:

$T_{\mathrm{i}}=$ template points

$S_{i}=$ signal points to be processed

$\bar{T}=$ mean of the template points

$\bar{S}=$ mean of the signal points

$N=$ number of points in the template

$\rho=$ the index of merit. 
This definition requires that $-1 \leq \rho \leq+1$. Identical signals have a value of +1 , whereas inverse signals have a value of -1 . The correlation coefficient is independent of baseline and amplitude differences between the template and the waveform under analysis. In order to increase the computational speed, by avoiding the square root operation, a signed $\rho^{2}$ was calculated:

$$
\eta=\operatorname{sign}(\rho) \rho^{2}
$$

and used as a discriminant. This performance measure $(\eta)$ was calculated, using the signal averaged sinus rhythm ventricular depolarization as a template for each lead configuration (unipolar and bipolar), and for all subsequent depolarizations in sinus rhythm and ventricular tachycardia.

A patient-specific window size was chosen to define the ventricular depolarization portion of the waveform alone and to exclude local repolarization and injury current caused by the temporary catheter. ${ }^{16-18}$ The end of depolarization was defined as the instant at which repolarization appeared to begin using the bipolar electrogram. This bipolar location was imposed on the unipolar signal as well. The beginning of depolarization was defined as the first deviation from baseline, separately for each lead configuration. Thus, separate window sizes were possible for each of the two electrode configurations within a single patient.

A software trigger was used to determine the position of ventricular depolarizations. This trigger $^{15}$ consists of a digital differentiator to determine the presence of ventricular depolarization. The method addresses possible trigger jitter ${ }^{19}$ by finding the largest $\eta$ within an 11 -ms window surrounding the initial point specified by the differentiator. The maximum computed $\eta$ within the window was reported as the value for each depolarization, and the range and mean of values for each analyzed passage were reported. Irregular ventricular depolarizations during sinus rhythm, due to premature atrial or ventricular depolarizations not eliminated by the automatic triggering process, were removed from the analysis manually.

It has been recently observed that the values derived from correlation waveform analysis of bipolar intraventricular electrograms do not have a
Gaussian distribution. ${ }^{18}$ Therefore, correct identification of ventricular tachycardia was defined in this study as complete separation of all $\eta$ values of ventricular tachycardia electrograms from all $\eta$ values of sinus rhythm electrograms. A single correlation value ( $\eta$ from Equation 2) of one class (sinus rhythm) which fell within the range of the second class (ventricular tachycardia), or vice versa, was considered a failure to discriminate between the two rhythms.

A separate calculation of the amount of separation $(\Delta)$ between ventricular tachycardia and sinus rhythm for each patient and lead configuration was performed. This measure was defined by:

$$
\Delta=\eta_{S R \min }-\eta_{V T \max }
$$

where $\eta_{S R \text { min }}$ was the minimum depolarization $\eta$ for sinus rhythm and $\eta_{V T \text { max }}$ was the maximum depolarization $\eta$ for ventricular tachycardia. For each patient, the amount of separation with unipolar analysis $\left(\Delta_{\text {uni }}\right)$ and bipolar analysis $\left(\Delta_{\mathrm{bi}}\right)$ was calculated. The number of patients in which $\Delta_{\text {uni }}$ was greater than $\Delta_{\mathrm{bi}}$ and vice versa was tabulated to give an additional measure of success for each lead configuration.

\section{Results}

Fourteen of 15 patients (93\%) had complete separation of $\eta$ ranges for unipolar and 14/15 for bipolar electrode configuration (Table I). Thirteen of the 14 absolute range separations were common to both groups. One tachycardia detection was successful using the bipolar lead configuration, but unsuccessful using the unipolar lead configuration. One other ventricular tachycardia detection was successful using the unipolar configuration, but unsuccessful using the bipolar lead configuration. Figure 1 summarizes the quantitative $\eta$ means and ranges for sinus rhythm and ventricular tachycardia.

Of the patients studied, $8 / 15$ (53\%) had greater separation between sinus rhythm and ventricular tachycardia waveforms using bipolar electrogram analysis $\left(\Delta_{\mathrm{bi}}>\Delta_{\mathrm{uni}}\right)$, while $7 / 15(43 \%)$ had greater separation between sinus rhythm and ventricular tachycardia waveforms using unipolar electrogram analysis $\left(\Delta_{\text {uni }}>\Delta_{\text {bi }}\right)$. 


\section{PATIENT}

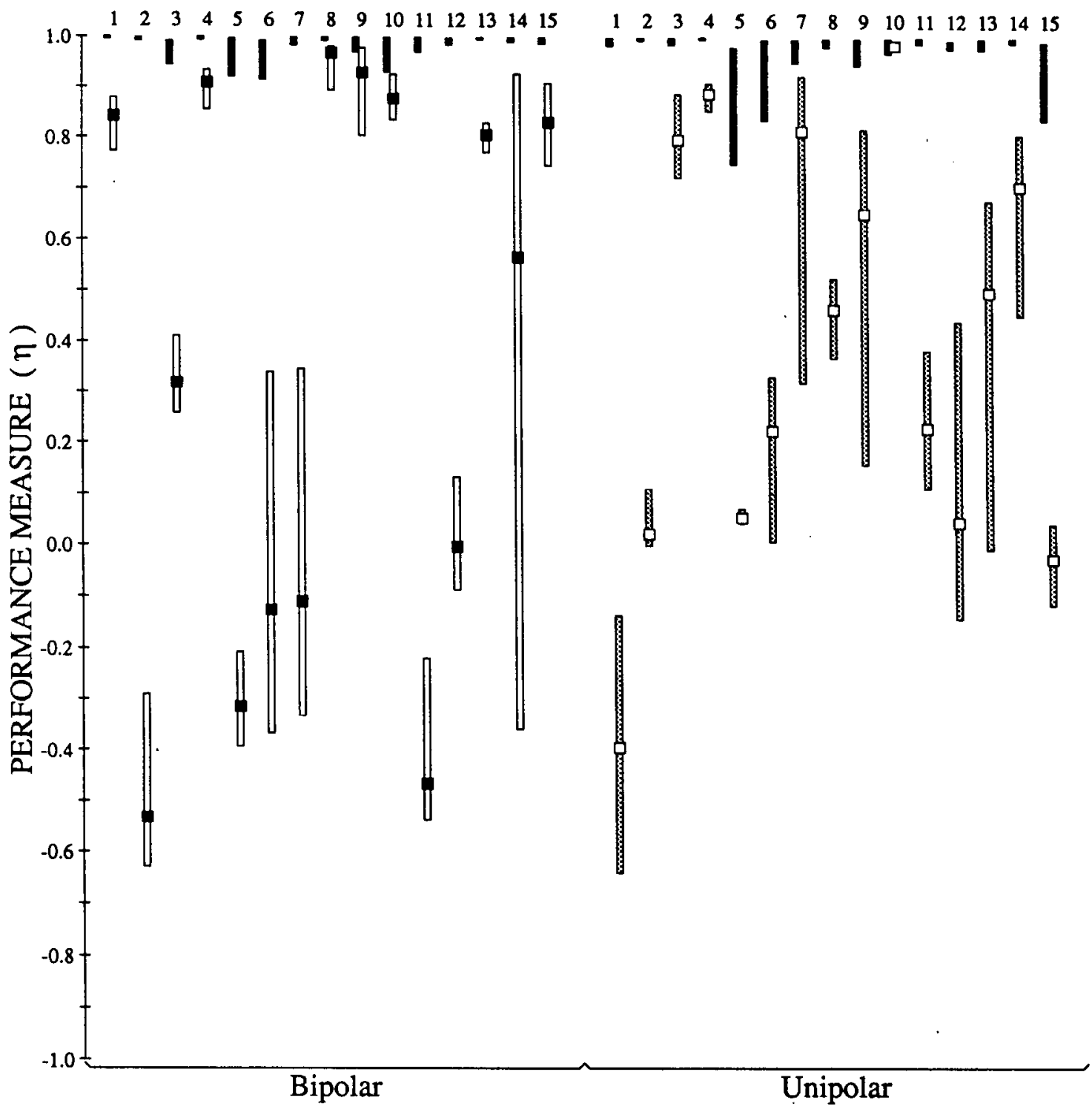

Figure 1. Results of the performance measure $(\eta)$ for discrimination of sinus rhythm from ventricular tachycardia using bipolar and unipolar electrograms. Patients are listed by number on the horizontal axis, on the left for bipolar, and on the right for unipolar electrograms. Ranges for sinus rhythm are indicated by solid black bars in each case. Means are not indicated on the sinus rhythm bars because the narrow range present in the majority of cases prohibit graphical display. Immediately below the sinus rhythm ranges (for all patients), ranges and means for ventricular tachycardia with the bipolar electrogram are indicated on the leftmost portion of the figure by unfilled bars and black squares, respectively. Ranges and means for ventricular tachycardia with the unipolar electrogram are shown on the rightmost portion of the figure by cross-hatched bars and unfilled squares, respectively. There is range overlap in patient 9 for the bipolar electrogram and in patient 10 for the unipolar electrogram. 


\section{Discussion}

The results of the present study suggest that correlation waveform analysis of unipolar and bipolar electrograms is equally effective in distinguishing ventricular tachycardia from sinus rhythm in supine patients at rest. In selected cases, differentiation between these two rhythms may be possible only with unipolar or bipolar electrogram analysis alone. Our results demonstrate that greater separation of the range of values $(\Delta)$ generated by correlation waveform analysis of sinus rhythm and ventricular tachycardia electrograms may occur with unipolar electrogram analysis in some patients while greater separation may occur with bipolar analysis in others.

Although the power demands of correlation waveform analysis, via more extensive hardware and software, exceed the capabilities of present implantable devices, the technique was used as a standard by which unipolar and bipolar waveform morphology could be compared independent of baseline and amplitude differences. The technique has previously been shown to discriminate well between ventricular tachycardia and sinus rhythm. ${ }^{14-16}$ More recently, the "Bin Area Method"16 and "Derivative Area Method,"20 which reduce the number of computations significantly from correlation and approach the expected capabilities of implantable devices, have been shown to discriminate with an accuracy equivalent to correlation.

Given the wide variety of commercially available bipolar sensing electrode configurations used with implantable devices (i.e., differences in electrode spacing, surface area, and materials), a bipolar electrode catheter typically used during electrophysiological study was tested. It remains unknown whether a smaller or larger spacing would optimize the electrogram morphologic differences between ventricular tachycardia and sinus rhythm. This study does not suggest an ideal electrode configuration. Rather, it compares a typical bipolar and an experimentally devised unipolar configuration. The unipolar indifferent electrode is positioned to simulate the location of an implantable cardioverter defibrillator. Our results suggest that the ability of either to better distinguish ventricular tachycardia from sinus rhythm may be patient dependent. Further work is nec- essary to determine ideal bipolar electrode spacings and surface areas for detection of ventricular tachycardia.

Identification of the critical electrogram features that distinguish individual intracardiac electrograms from one another has been elusive. Bipolar electrograms are formed from the potential difference of two unipolar electrograms. It might be anticipated, therefore, that information critical to the "uniqueness" of any recorded ventricular activation wave front might be lost in the subtraction process. The results of the present study, however, demonstrate that this is not necessarily the case when discriminating ventricular tachycardia from sinus rhythm electrograms.

Previously proposed methods for detecting ventricular tachycardia using ventricular endocardial electrograms have examined several isolated morphologic features including the first derivative of the electrogram, the ratio of the peak electrogram amplitude to electrogram cycle length, the ratio of peak positive to peak negative electrogram amplitude, the maximum slope (dV/ $\mathrm{dt}$ ), and the phase angle between time-delayed recordings of the same electrogram. ${ }^{21-23}$ These methods have been tested on a limited number of selected patients and have not been uniformly successful.

One other study has compared the results of analysis of the "area of difference" between a smaller number of unfiltered bipolar and unipolar electrograms recorded during sinus rhythm and induced ventricular tachycardia in 10 patients. ${ }^{24}$ Although this method has the potential disadvantage of depending upon electrogram amplitude and baseline fluctuations, some of which may be artifactual, ventricular tachycardia was successfully discriminated from sinus rhythm in all cases. High pass filtering and limiting of the sample window width had a negative impact upon the ability of this method to discriminate ventricular tachycardia from sinus rhythm, however, suggesting that low frequency and far-field portions of the electrogram might be important for rhythm discrimination. Of note was the greater negative impact these limitations had upon the bipolar as compared to unipolar accuracy of the "area of difference" method. 
In contrast, the findings of the present study suggest that maximum discrimination between sinus rhythm and ventricular tachycardia is not necessarily determined by electrogram configuration alone. This suggests that other features, for example the velocity and spatial orientation of the ventricular activation wave front relative to electrode location, may be among the more important

\section{References}

1. Fisher JD, Goldstein M, Ostrow E, et al. Maximal rate of tachycardia development: Sinus tachycardia with sudden exercise vs. spontaneous ventricular tachycardia. PACE 1983; 6:221-228.

2. Geibel A, Zehender M, Brugada P, et al. Changes in cycle length at the onset of sustained tachycardias-Importance for antitachycardia pacing. Am Heart J 1988; 588-592.

3. Fromer M, Kus T, Dubuc M, et al. Oscillation of ventricular tachycardia cycle lengths. (abstract) PACE 1987; 10:451.

4. Nathan AW, Creamer JE, Davies DW, et al. Clinical experience with a new versatile, software based, tachycardia reversion pacemaker. (abstract) J Am Coll Cardiol 1986; 7:184A.

5. Olson WH, Bardy GH. Cycle length and morphology at the onset of spontaneous ventricular tachycardia and fibrillation. (abstract) PACE 1986; 9:284.

6. Olson W, Bardy G, Mehra R, et al. Comparison of different onset and stability algorithms for detection of spontaneous ventricular arrhythmias. (abstract) PACE 1987; 10:439.

7. Olson W, Bardy G, Mehra R, et al. Onset and stability for ventricular tachycardia detection in an implantable pacer-cardioverter-defibrillator. Computers in Cardiology 1987; 167-170.

8. Tomaselli G, Scheinman M, Griffin J. The utility of timing algorithms for distinguishing ventricular from supraventricular tachycardias. (abstract) PACE 1987; 10:415.

9. Warren J, Martin RO. Clinical evaluation of automatic tachycardia diagnosis by an implanted device. (abstract) PACE 1986; 4:16.

10. Ripley KL, Bump TE, Arzbaecher RC. Evaluation of techniques for recognition of ventricular arrhythmias by implanted devices. IEEE Trans Biomed Eng 1989; 36:618-624.

11. Echt DS, Armstrong K, Schmidt P, et al. Clinical experience, complications and survival in 70 patients with the automatic implantable cardioverter/defibrillator. (abstract) Circulation 1984; 71:289.

12. Echt DS. Potential hazards of implanted devices for the electrical control of tachyarrhythmias. PACE 1984; 7:580-587.

13. Steinberg JS, Sugalski JS, Haratonic K, et al. Cardiac rhythm precipitating automatic implantable determinants in the assessment of intracardiac electrogram morphology.

Acknowledgements: The authors wish to thank Colleen Hoover, B.S., Barbara Nagrant, R.C.V.T., and Judy Jaffke, R. T.(R), for their technical assistance in the Cardiac Electrophysiology Laboratory and Ms. Debbie Laird for her assistance in preparation of this manuscript.

cardioverter defibrillator discharges in outpatients: Observations from transtelephonic recordings. (abstract) Circulation 1989; 80:530.

14. Lin D, DiCarlo LA, Jenkins JM. Identification of ventricular tachycardia using intracavitary ventricular electrograms: Analysis of time and frequency domain patterns. PACE 1988; 11:15921606.

15. Throne RD, Jenkins JM, Winston SA, et al. Discrimination of retrograde from anterograde atrial activation using intracardiac electrogram waveform analysis. PACE 1989; 12:1622-1630.

16. Throne RD, Jenkins JM, DiCarlo LA. The bin area method: A computationally efficient technique for analysis of ventricular and atrial intracardiac electrograms. PACE 1990; 13:1286-1297.

17. Throne RD, DiCarlo LA, Jenkins JM, et al. Paroxysmal bundle branch block of supraventricular origin: A possible source of misdiagnosis in detecting ventricular tachycardia using time domain analyses of intraventricular electrograms. PACE $1990 ; 13: 453-468$.

18. Throne RD, Jenkins JM, DiCarlo LA. Intraventricular electrogram analysis for ventricular tachycardia detection: Statistical validation. PACE 1990; 13:1596-1601.

19. Rompleman O, Ros HH. Coherent averaging technique: A tutorial review. Parts 1 and 2. J Biomed Eng 1986; 8:24-35.

20. Throne RD, Jenkins JM, Winston SA, et al. Derivative area method: A new technique for detecting ventricular tachycardia. (abstract) Circulation 1989; 80:II-658.

21. Davies DW, Wainwright RJ, Tooley MA, et al. Detection of pathological tachycardia by analysis of electrogram morphology. PACE 1986; 9:200208.

22. Pannizzo F, Wanliss M, Furman S, et al. Discriminator of ventricular tachycardia electrograms by syntactic methods. (abstract) J Am Coll Cardiol 1987; 9:98A.

23. Tronstad A, Hoff PI, Ohm OJ. A new method for automatic detection of ventricular tachyarrhythmias. (abstract) PACE 1987; 10:415.

24. Langberg JL, Gibb WJ, Auslander DM, et al. Identification of ventricular tachycardia with use of the morphology of the endocardial electrogram. Circulation 1988; 77:1363-1369. 
Copyright of Pacing \& Clinical Electrophysiology is the property of Blackwell Publishing Limited and its content may not be copied or emailed to multiple sites or posted to a listserv without the copyright holder's express written permission. However, users may print, download, or email articles for individual use. 